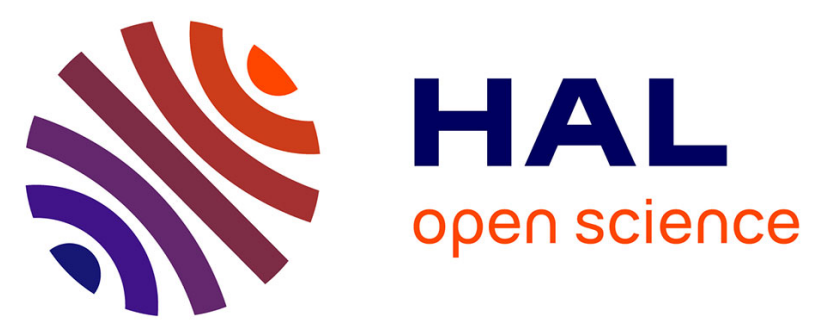

\title{
Loss of two families of SPX domain-containing proteins required for vacuolar polyphosphate accumulation coincides with the transition to phosphate storage in green plants
}

Long Wang, Xianqing Jia, Yuxin Zhang, Lei Xu, Benoît Menand, Hongyu Zhao, Houqing Zeng, Liam Dolan, Yiyong Zhu, Keke Yi

\section{To cite this version:}

Long Wang, Xianqing Jia, Yuxin Zhang, Lei Xu, Benoît Menand, et al.. Loss of two families of SPX domain-containing proteins required for vacuolar polyphosphate accumulation coincides with the transition to phosphate storage in green plants. Molecular Plant, 2021, 10.1016/j.molp.2021.01.015 . cea-03125841

\section{HAL Id: cea-03125841 https://hal-cea.archives-ouvertes.fr/cea-03125841}

Submitted on 7 Oct 2021

HAL is a multi-disciplinary open access archive for the deposit and dissemination of scientific research documents, whether they are published or not. The documents may come from teaching and research institutions in France or abroad, or from public or private research centers.
L'archive ouverte pluridisciplinaire HAL, est destinée au dépôt et à la diffusion de documents scientifiques de niveau recherche, publiés ou non, émanant des établissements d'enseignement et de recherche français ou étrangers, des laboratoires publics ou privés. 


\title{
Loss of two families of SPX domain-containing proteins required for vacuolar polyphosphate accumulation coincides with the transition to phosphate storage in green plants
}

\author{
Long Wang ${ }^{1,2,6}$, Xianqing Jia ${ }^{1,6}$, Yuxin Zhang ${ }^{1}$, Lei Xu ${ }^{1}$, Benoit Menand ${ }^{3}$, Hongyu Zhao ${ }^{1}$, \\ Houqing Zeng ${ }^{4}$, Liam Dolan ${ }^{5}$, Yiyong $\mathrm{Zhu}^{2,{ }^{*}}$ and Keke $\mathrm{Yi}^{1, *}$ \\ ${ }^{1}$ Key Laboratory of Plant Nutrition and Fertilizers, Ministry of Agriculture and Rural Affairs, Institute of Agricultural Resources and Regional Planning, Chinese \\ Academy of Agricultural Sciences, Beijing 100081, China \\ ${ }^{2}$ Jiangsu Provincial Key Lab for Organic Solid Waste Utilization, National Engineering Research Center for Organic-based Fertilizers, Jiangsu Collaborative \\ Innovation Center for Solid Organic Waste Resource Utilization, Nanjing Agricultural University, Nanjing 210095, China \\ ${ }^{3}$ Aix Marseille Univ, CEA, CNRS, BIAM, Luminy Plant Genetics and Biophysics Team, Marseille 13009, France \\ ${ }^{4}$ College of Life and Environmental Sciences, Hangzhou Normal University, Hangzhou 311121, China \\ ${ }^{5}$ Gregor Mendel Institute of Molecular Plant Biology GmbH, Dr. Bohr-Gasse 3, 1030 Vienna, Austria \\ ${ }^{6}$ These authors contributed equally to this article. \\ *Correspondence: Yiyong Zhu (yiyong1973@njau.edu.cn), Keke Yi (yikeke@gmail.com) \\ https://doi.org/10.1016/j.molp.2021.01.015
}

\section{ABSTRACT}

Phosphorus is an essential nutrient for plants. It is stored as inorganic phosphate (Pi) in the vacuoles of land plants but as inorganic polyphosphate (polyP) in chlorophyte algae. Although it is recognized that the SPXMajor Facilitator Superfamily (MFS) and VPE proteins are responsible for Pi influx and efflux, respectively, across the tonoplast in land plants, the mechanisms that underlie polyP homeostasis and the transition of phosphorus storage forms during the evolution of green plants remain unclear. In this study, we showed that CrPTC1, encoding a protein with both SPX and SLC (permease solute carrier 13) domains for Pi transport, and CrVTC4, encoding a protein with both SPX and vacuolar transporter chaperone (VTC) domains for polyP synthesis, are required for vacuolar polyP accumulation in the chlorophyte Chlamydomonas reinhardtii. Phylogenetic analysis showed that the SPX-SLC, SPX-VTC, and SPX-MFS proteins were present in the common ancestor of green plants (Viridiplantae). The SPX-SLC and SPX-VTC proteins are conserved among species that store phosphorus as vacuolar polyP and absent from genomes of plants that store phosphorus as vacuolar Pi. By contrast, SPX-MFS genes are present in the genomes of streptophytes that store phosphorus as $\mathrm{Pi}$ in the vacuoles. These results suggest that loss of SPX-SLC and SPX-VTC genes and functional conservation of SPX-MFS proteins during the evolution of streptophytes accompanied the change from ancestral polyP storage to Pi storage.

Key words: Chlamydomonas, polyphosphate, SPX-SLC, SPX-VTC, vacuolar phosphate, plant evolution

Wang L., Jia X., Zhang Y., Xu L., Menand B., Zhao H., Zeng H., Dolan L., Zhu Y., and Yi K. (2021). Loss of two families of SPX domain-containing proteins required for vacuolar polyphosphate accumulation coincides with the transition to phosphate storage in green plants. Mol. Plant. 14, 838-846.

\section{INTRODUCTION}

Phosphorus is a finite non-renewable resource essential for life on earth. It is a critical component of nucleic acids, phospholipids, and ATP, and participates in numerous biochemical pathways, including gene expression and signal transduction. Consequently, phosphorus deficiency can impair a wide range of biological processes, ultimately affecting plant growth and development
(Raghothama, 1999). To cope with the stress caused by phosphorus deficiency, green plants have evolved adaptive mechanisms for phosphorus acquisition and conservation that enhance survival where available phosphorus is limiting.

Published by the Molecular Plant Shanghai Editorial Office in association with Cell Press, an imprint of Elsevier Inc., on behalf of CSPB and CEMPS, CAS. 
Extant green plants (Viridiplantae) comprise two monophyletic lineages, Chlorophyta and Streptophyta (land plants and their closest algal relatives, a grade collectively known as streptophyte algae) (Becker and Marin, 2009). Vascular land plants take up phosphorus as the oxyanion phosphate $(\mathrm{Pi})$ from soil through roots and associated fungal symbionts. However, nearly $70 \%$ of global arable land is deficient in $\mathrm{Pi}$, and land plants have evolved multiple strategies to adapt to this stress. A class of proteins that exclusively contain an SPX (SYG1/Pho81/XPR1) domain (SPXs) are the main negative regulators of $\mathrm{Pi}$ signaling. They can modulate the activity of central transcription factors, the PHOSPHATE STARVATION RESPONSE (PHR) proteins, in plants (Lv et al., 2014; Puga et al., 2014; Wang et al., 2014). Other proteins with conserved SPX domains also regulate $\mathrm{Pi}$ transport and signaling. PHOSPHATE1 (PHO1), an SPX-EXS protein with both SPX and EXS (ERD1, XPR1, SYG1) domains, is a transporter required for $\mathrm{Pi}$ loading into root xylem vessels that facilitates $\mathrm{Pi}$ translocation from roots to shoots (Poirier et al., 1991; Hamburger et al., 2002). The SPX-MFS (major facilitator superfamily) proteins, which contain both SPX and MFS domains, transport Pi into the vacuole (Liu et al., 2015, 2016; Xu et al., 2019). A class of E3 ligases (NLAs) with both SPX and RING (Really Interesting Gene) domains are involved in the degradation of Phosphate Transporter 1 proteins (Park et al., 2014). Taken together, these lines of evidence demonstrate that SPX-domain-containing proteins are involved in phosphate transport and phosphorus signaling in land plants, but nothing is known about the function of SPX-containing proteins in algae.

In Chlamydomonas reinhardtii (Chlorophyta), phosphorus is stored in vacuoles in the form of dense, calcium-associated polymers of phosphate (polyP) bodies that are called acidocalcisomes (Ruiz et al., 2001). PolyP is a linear, unbranched polymer of three to several hundred $\mathrm{Pi}$ residues linked by phosphoanhydride bonds. PolyP has been observed in the vacuole of the red alga Cyanidioschyzon merolae and in a number of other chlorophyte algae (Yagisawa et al., 2009; Lorenzo-Orts et al., 2020). Vacuolar polyP is synthesized in the cytoplasm and transported into the vacuole by the VTC (vacuolar transporter chaperone) complex (Hothorn et al., 2009). Consistent with the role of the VTC complex in polyP synthesis, a Chlamydomonas mutant with a VTC1 deletion accumulates less polyP and has fewer acidocalcisomes than the wild type (WT) (Aksoy et al., 2014). Vtc4p (a protein with both SPX and VTC domains), one of the VTC subunits, is a polyP polymerase in yeast (Hothorn et al., 2009), and its Chlamydomonas homolog (VTC4) was suggested to be required for polyP synthesis (Aksoy et al., 2014). However, the regulation of vacuolar polyP accumulation in chlorophyte algae remains unclear. Unlike chlorophytes, land plants store phosphorus as $\mathrm{Pi}$ in their vacuoles (Lorenzo-Orts et al., 2020). However, the evolution of vacuolar phosphorus storage forms in the Viridiplantae is still unknown.

To examine the evolution of vacuolar phosphorus storage forms in Viridiplantae, we showed that phosphorus is stored as polyphosphate (polyP) in chlorophyte vacuoles; furthermore, we characterized a new vacuolar Phosphate Transporter $C$ in C. reinhardtii (termed CrPTC1), one of the previously uncharacterized SPX-SLC proteins, which has both SPX and SLC (permease solute carrier 13) domains. Our results suggest that CrPTC1 catalyzes $\mathrm{Pi}$ transport out of acidocalcisomes and is involved in polyP accumulation and $\mathrm{Pi}$ starvation-dependent signaling in Chlamydomonas. We further showed that CrVTC4, a protein with both SPX and VTC domains, is essential for polyP synthesis. We performed a phylogenetic analysis to identify which SPX domain-containing proteins were present in the last common ancestor of chlorophytes and streptophytes, which existed approximately 1200 million years ago (Nie et al., 2020). We showed that the SPX-SLC and SPX-VTC proteins are ancient and conserved in the genomes of plants that store phosphorus as vacuolar polyP. However, they are not present in the genomes of later-diverging streptophytes that store phosphorus as vacuolar Pi but not as polyP. The conservation of SPX-MFS and the loss of SPX-VTC and SPX-SLC in streptophyte algae accompanied the change in vacuolar phosphorus storage from polyP to $\mathrm{Pi}$ and may have been a pre-adaptation for land colonization.

\section{RESULTS AND DISCUSSION}

\section{The Chlamydomonas SPX-SLC protein CrPTC1 is a $\mathrm{Pi}$} transporter involved in polyP storage

Given the function of proteins with SPX domains in Pi signaling and transport in land plants, we searched the $C$. reinhardtii genome for sequences that encoded proteins with SPX domains. One of these proteins, CrPTC1, also harbored an anionpermease SLC domain, suggesting that it may have a function in ion transport. Topology prediction showed that CrPTC1 had 14 transmembrane segments with an N-terminal SPX domain that was predicted to be located in the cytoplasm (Figure 1A). To test whether CrPTC1 transported Pi across membranes, we expressed CrPTC1 in a yeast mutant strain (YP100) that is defective in $\mathrm{Pi}$ transport and does not grow unless supplemented with galactose (Figure 1B). CrPTC1 restored growth on a medium that contained $20 \mathrm{mM} \mathrm{Pi}$, where it grew almost as well as yeast that expressed the high-affinity Pi transporter $\mathrm{PHO} 84$. Some growth occurred on medium that contained $100 \mu \mathrm{M} \mathrm{Pi}$, although it was less than that of yeast that expressed PHO84 (Figure 1B). The growth of YP100 transformed with CrPTC1 indicated that net Pi uptake occurred in yeast when CrPTC1 was expressed, consistent with the hypothesis that CrPTC1 is a Pi transporter. Further analysis showed that oocytes expressing CrPTC1 took up Pi at $\sim 0.1 \mathrm{nmol} \mathrm{h}^{-1}$ per oocyte, whereas a water-injected control exhibited lower Pi uptake of $\sim 0.01 \mathrm{nmol} \mathrm{h}^{-1}$ per oocyte (Supplemental Figure 1). This result verified the role of CrPTC1 as a Pi transporter.

To investigate the function of CrPTC1, we characterized two mutant alleles (Crptc1-1 and Crptc1-2) (Supplemental Figure 2A) isolated from a library of Chlamydomonas indexed insertional mutants (Li et al., 2019). We mainly used Crptc1-1 for analysis because it is a complete loss-of-function (null) mutant, whereas Crptc1-2 is a hypomorph (incomplete loss-of-function) mutant (Supplemental Figure 2B-2E). Our results showed that total phosphorus content was higher in the Crptc1-1 mutant than in the WT (Figure 1B). The defect in total phosphorus accumulation of Crptc1-1 was suppressed in the CrPTC1 complementation line (Crptc1-1:PTC1-13) and was partially suppressed in the line Crptc1-1:PTC1-16, confirming that the Crptc1 mutant phenotype was caused by the disruption of 
A

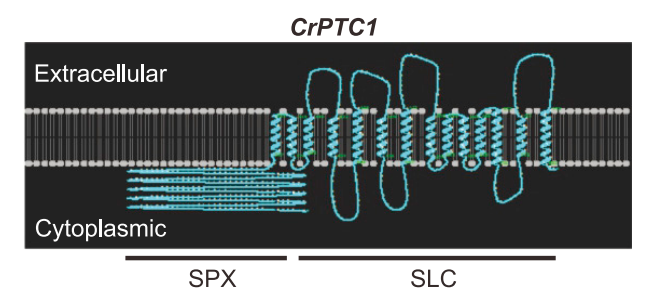

B

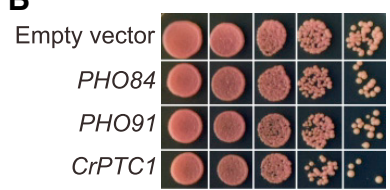

YNB-Galactose

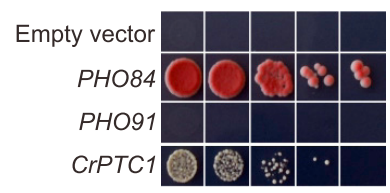

YNB-P + $1 \mathrm{mM} \mathrm{Pi}$

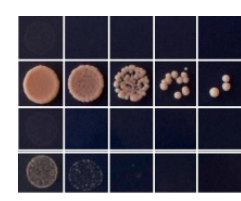

YNB-P + $0.1 \mathrm{mM} P$

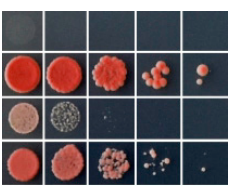

YNB-P + $20 \mathrm{mM}$ Pi
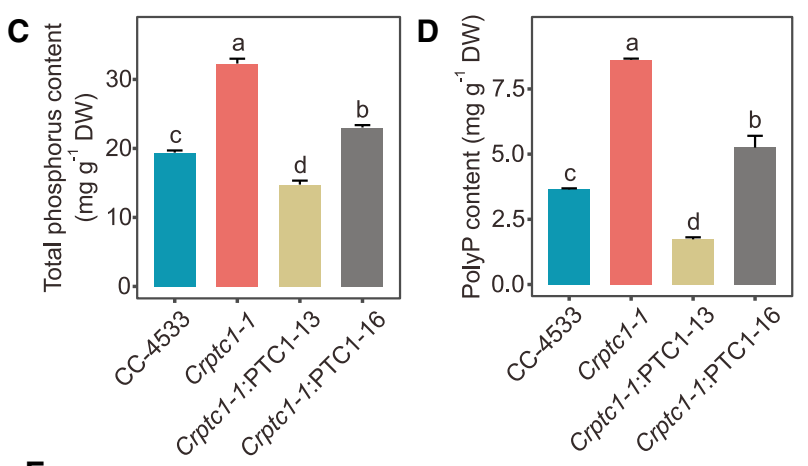

E

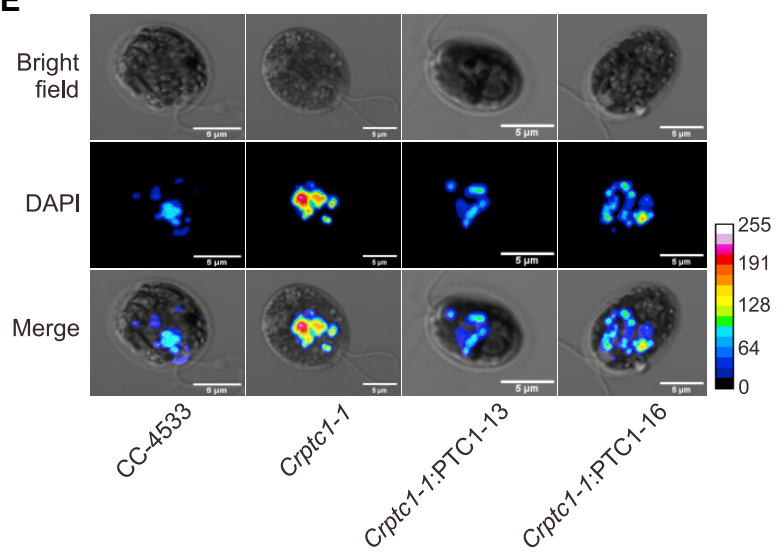

Figure 1. The Chlamydomonas Crptc1 mutant over-accumulates total phosphorus and polyP.

(A) Predicted topological structure of CrPTC1 showing the transmembrane SLC domain and the SPX domain.

(B) CrPTC1 (SPX-SLC in C. reinhardtii) mediates Pi membrane transport in yeast. Complementation of yeast mutant YP100 by yeast ScPHO84, SCPHO91, and Chlamydomonas PTC1 driven by the ADH1 promoter.

(C) Total phosphorus content of the WT CC-4533, the Crptc1-1 mutant, and two complementation strains.

(D) PolyP content of the WT CC-4533, the Crptc1-1 mutant, and two complementation strains. Error bars indicate SE. Differences were tested by ANOVA using the least significant difference (LSD) method with a Bonferroni correction at $\alpha=0.05$. Means with the same letters are not significantly different. (E) Representative pseudo-color images of cellular polyP stained with DAPI. DAPI was excited at $405 \mathrm{~nm}$ and an emission spectrum of 532-632 nm was collected using a ZEISS LSM 880 scanning confocal microscope. Bar, $5 \mu \mathrm{m}$. Experiments were repeated three times with similar results.

CrPTC1 gene function (Figure $1 \mathrm{C}$ and Supplemental Figure 2D and 2E). We further noticed that polyP content in both Crptc1-1 and Crptc1-2 mutants was significantly higher than in WT; in particular, the Crptc1-1 mutant accumulated approximately three times more polyP than the WT (Figure 1D and Supplemental Figure 2F). The higher accumulation of polyP in the mutant was significantly suppressed in the complementation lines (Figure 1D).

To determine whether vacuolar polyP accumulation is defective in the Crptc1-1 mutant, we stained polyP in Chlamydomonas cells. Consistent with the report that most of the polyP in the Chlamydomonas cell accumulates in acidocalcisomes (Komine et al., 2000), we detected a punctate polyP fluorescence signal that colocalized with acidic compartments indicated by LysoTracker Green (Supplemental Figure 3). DAPI fluorescence was higher in the Crptc1-1 mutant than in the WT, and this enhanced fluorescence signal was suppressed by the introduction of a WT copy of CrPTC1 (Figure 1E). This result suggests that CrPTC1 is a tonoplast Pi transporter that facilitates Pi release from acidocalcisomes. To test this hypothesis, we attempted to express a CrPTC1-GFP fusion in Chlamydomonas. However, after 10 attempts at transformation and screening of more than 2000 zeocin-resistant clones, we failed to isolate GFP-positive colonies. Although we could not determine the subcellular localization of CrPTC1 in Chlamydomonas, we were able to detect the vacuolar membrane localization of CrPTC1-GFP in yeast cells (Supplemental Figure 4). Together, these findings indicated that CrPTC1 may be an acidocalcisomal membrane protein that facilitates Pi release from acidocalcisomes.

\section{Disruption of CrPTC1 causes defective Pi starvation- dependent signaling}

The observation that levels of total phosphorus and polyP are higher in CrPTC1 loss-of-function mutants than in the WT is consistent with the hypothesis that CrPTC1 is involved in Pi starvationdependent signaling. To test this hypothesis, we first measured the activity of alkaline phosphatase, which is secreted by Chlamydomonas under $\mathrm{Pi}$ starvation but not in Pi-replete conditions (Wykoff et al., 1999). Under Pi-sufficient conditions, extracellular phosphatase activity was barely detectable in the WT, the Crptc1-1 mutant, and the complementation strains (Figure 2A and Supplemental Figure 5). However, under phosphate starvation, the Crptc1-1 mutant accumulated higher levels of extracellular phosphatase than the WT and the complementation strains, as indicated by the intense blue precipitate surrounding 
A

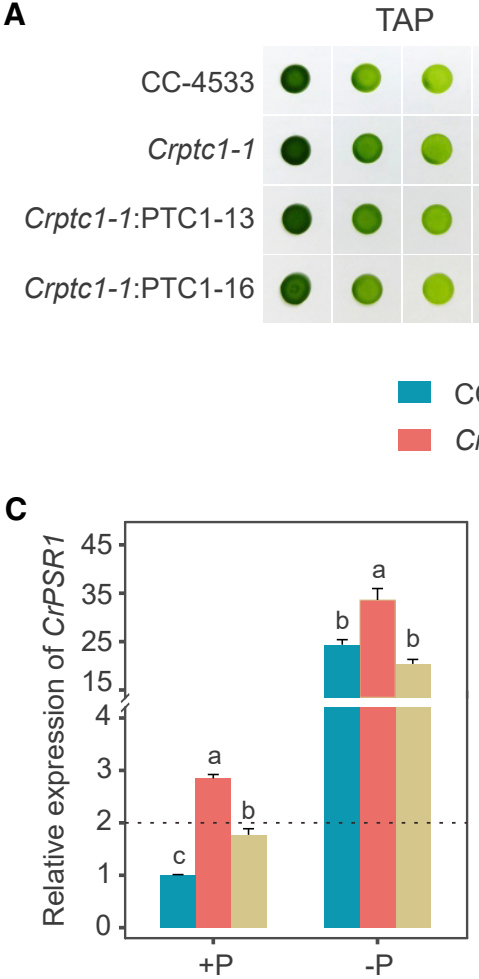

TA

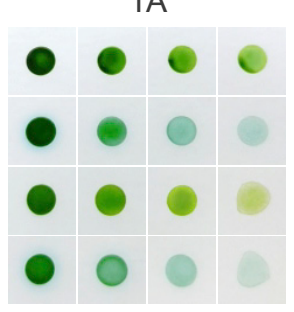

Crptc1-1:PTC1-13

Crptc1-1:PTC1-16

\begin{abstract}
D
\end{abstract}

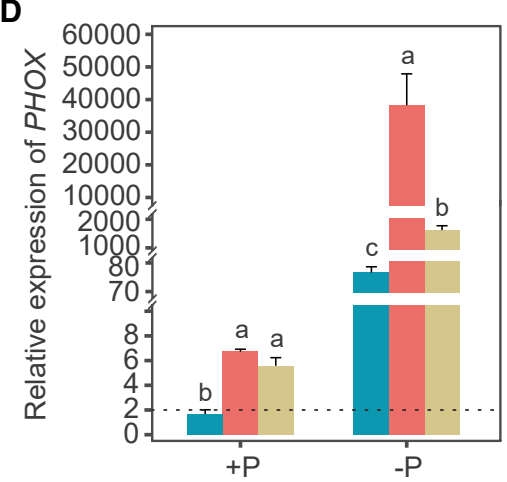

B

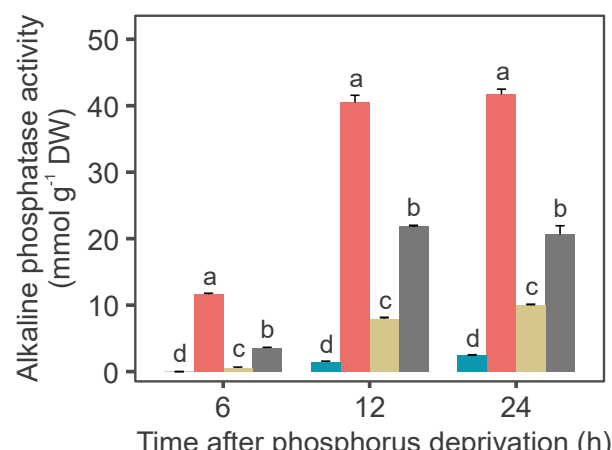

\section{E}

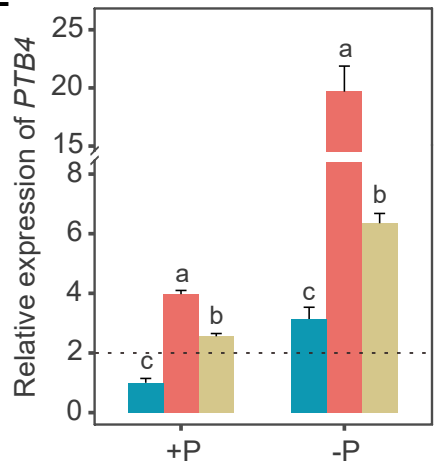

Figure 2. Disruption of CrPTC1 caused an alteration in Pi starvation-dependent signaling.

(A) Qualitative analysis of phosphatase activity of WT CC-4533, the Crptc1-1 mutant, and complementation strains. Cells were streaked onto TAP and TA ([tris-acetate-phosphate] medium without phosphate) solid medium prior to spraying the plates with the colorimetric phosphatase substrate 5-bromo-4chloro-3-indolyl-P, which turns blue after cleavage by alkaline phosphatase. The plates were allowed to develop for 2 days before recording the results. (B) Alkaline phosphatase activity of WT CC-4533, the Crptc1-1 mutant, and complementation strains after 6, 12, or $24 \mathrm{~h}$ of phosphorus deprivation. Alkaline phosphatase activity is represented as absorbance at $410 \mathrm{~nm}$ of $\mathrm{p}$-nitrophenol produced per gram of algal dry weight.

(C-E) Quantification of PSR1 (C), PHOX (D), and PTB4 (E) mRNA by qRT-PCR over a period of $6 \mathrm{~h}$ of phosphate deprivation. CBLP was used as the internal control. The data are presented relative to the values of CC-4533 under +P conditions. Error bars indicate SE. Differences were tested by ANOVA using the LSD method with a Bonferroni correction at $\alpha=0.05$. Means with the same letters are not significantly different.

the colonies (Figure 2A and Supplemental Figure 5). We next measured the phosphatase activity of these strains after $\mathrm{Pi}$ deprivation. Although phosphatase activity was barely detected in the WT after $6 \mathrm{~h}$ of Pi starvation, it increased after $12 \mathrm{~h}$ or $24 \mathrm{~h}$ of starvation (Figure 2B). Phosphatase activity was higher in the Crptc1-1 mutant than in the WT after $6 \mathrm{~h}$ of $\mathrm{Pi}$ starvation (Figure 2B), and this activity increased further after 12 or $24 \mathrm{~h}$ of Pideprivation, when levels were 40-fold higher than those observed in the WT (Figure 2B). Both the high extracellular phosphatase activity and the enhanced phosphatase activity in Crptc1-1 were fully or partially suppressed in the complementation strains (Figure $2 \mathrm{~A}$ and $2 \mathrm{~B}$ ). Together, these data suggest either that $\mathrm{Pi}$ starvation signaling is more active in the Crptc1-1 mutant than in the WT under Pi starvation stress or that Crptc1 mutants are more sensitive to Pi starvation stress than the WT.

We next measured steady-state transcript levels of genes that encode PHOX phosphatase, the PTB4 phosphate transporter, and the PSR1 transcription factor in the WT, the Crptc1-1 mutant, and a complementation strain (Crptc1-1:PTC1-13) grown with sufficient $\mathrm{Pi}$. Expression of PSR1, PHOX, and PTB4 was higher in the Crptc1-1 mutant than in the WT under Pi-replete conditions (Figure $2 \mathrm{C}-2 \mathrm{E}$ ), indicating that $\mathrm{Pi}$ starvation signaling was activated in the mutant under these conditions; steady-state levels of PHOX and PTB4 mRNA were much higher in the Crptc1-1 mutant than that in WT in Pi-replete conditions. Furthermore, steady-state levels of PSR1 mRNA were slightly but significantly higher in the Crptc1-1 mutant than in the WT. Higher levels of PSR1, PHOX, and PTB4 expression in Crptc1 were largely suppressed in the CrPTC1 complementation line (Figure 2C-2E). These data indicate that a Pi-stress response is constitutively active in the Crptc1-1 mutant, whereas this response is inducible in the WT. These data suggest that CrPTC1 represses the low $\mathrm{Pi}$-stress response.

Together, these data suggest that the SPX-SLC protein CrPTC1 modulates polyP accumulation by facilitating $\mathrm{Pi}$ release from the acidocalcisome and that loss of CrPTC1 function alters $\mathrm{Pi}$ starvation-dependent signaling in Chlamydomonas.

SPX-SLC and SPX-VTC are ancient and have been lost during the evolution of Streptophyta

To trace the phylogenetic history of SPX-containing proteins, we carried out a phylogenetic analysis of all SPX-containing proteins from 49 species of Archaeplastida, including 41 Viridiplantae species, three Glaucophyta algae, and five Rhodophyta algae (red algae). We generated alignments for the SPX domains and 
A

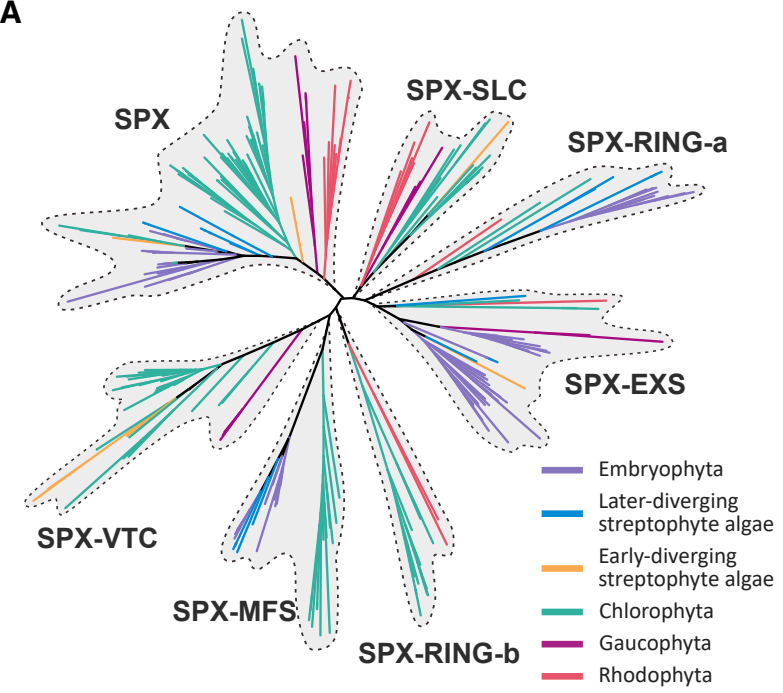

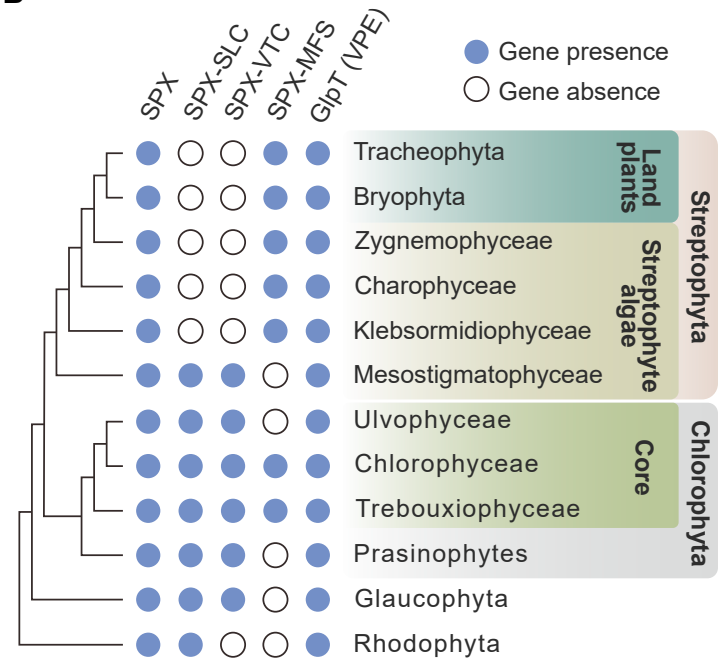

Figure 3. Evolution and presence of SPX domain-containing genes in the plant kingdom.

(A) Phylogeny of SPX domain-containing proteins in 49 species of plantae. M. viride was designated as an early-diverging streptophyte alga, and Klebsormidium nitens, C. braunii, and S. muscicola were late-diverging streptophytes. The tree was built from SPX domain sequences of all SPX domaincontaining proteins. Multiple sequence alignment was performed using MAFFT, and the tree was constructed using IQ-Tree with the maximum likelihood method. It has been submitted here: https://itol.embl.de/tree/20211940236266511594463324.

(B) Presence of SPX domain-containing genes and GIpT (VPE) genes in different phyla. SPX, SPX-SLC, SPX-VTC, SPX-MFS, SPX-EXS, SPX-RING, and GIpT (VPE) orthologs were retrieved from OneKP or genome database searches (Supplemental Table 1).

constructed trees using maximum likelihood statistics (Supplemental Data 1 and Supplemental Figures 6-11). The topology of the tree shows that SPX, SPX-SLC, SPX-VTC, SPXMFS, and SPX-EXS proteins each form distinct monophyletic clades. The SPX-RING proteins fall into two different monophyletic clades: one SPX-RING clade (designated SPX-RING-a) is sister to the SPX-EXS clade, and the second (designated SPXRING-b) is sister to the clade comprising SPX-MFS and SPXVTC proteins (Figure 3A). SPX-MFS may be the most recently evolved clade because it contains no members in either Rhodophyta or Glaucophyta, whereas the presence of SPX-MFS proteins in both Chlorophyta and Streptophyta suggests that they were present in the common ancestor of these lineages (Supplemental Figure 12). SPX-VTC proteins are present in Glaucophyta, Chlorophyta, and the early-diverging streptophyte algae Mesostigmatophyceae, indicating that they existed in the Archaeplastida lineage after the divergence of Rhodophyta. SPX-SLC proteins are present in Rhodophyta, Glaucophyta, Chlorophyta, and Mesostigmatophyceae, suggesting that they appeared earlier than the SPX-VTC proteins and were present in the common ancestor of the Archaeplastida. Interestingly, both SPX-SLC and SPX-VTC proteins are absent in the laterdiverging streptophytes, including streptophyte algae and Embryophyta (Figure 3B). This is consistent with the hypothesis that SPX-SLC and SPX-VTC proteins were present in the ancestors of the early-diverging streptophytes but lost in more derived groups of streptophytes. These data suggest that SPX-SLC and SPX-VTC proteins perform a function in the Archaeplastida that was lost in later-diverging streptophytes.

\section{SPX-VTC is essential for polyP synthesis}

VTC4, an SPX-VTC protein, is a polyP polymerase that is essential for vacuolar polyP synthesis in yeast (Hothorn et al., 2009).
Because genes encoding SPX-VTC proteins are present in the genomes of chlorophytes but not late-diverging streptophytes, we hypothesized that SPX-VTC proteins participate in acidocalcisomal polyP synthesis in Chlamydomonas and that neither Klebsormidium nor Chara braunii store acidocalcisomal polyP. To test this hypothesis, we first identified two CrVTC4 loss-of-function Chlamydomonas mutants and measured their polyP accumulation (Supplemental Figure 13). Our data showed that polyP was undetectable in the Crvtc4 mutants, indicating that CrVTC4 is essential for polyP synthesis. Furthermore, our in vitro synthesis assay showed that truncated CrVTC4 with the putative kinase domain could synthesize polyP, thereby verifying that CrVTC4 is a polyP polymerase (Supplemental Figure 13F and 13G).

We then examined vacuolar phosphorus storage forms in earlydiverging Mesostigma viride and later-diverging Klebsormidium and $C$. braunii. PolyP staining and data from the published literature (Pick and Weiss, 1991; Ruiz et al., 2001) showed that, like chlorophytes, Mesostigma accumulated polyP in vacuoles, whereas neither Klebsormidium nor $C$. braunii accumulated polyP in vacuoles (Figure $4 \mathrm{~A}$ and $4 \mathrm{~B}$ ). These data suggest that storage of $\mathrm{Pi}$ in the vacuole evolved in the streptophyte lineage after the divergence of Mesostigma but before the divergence of Klebsormidium.

\section{Evolution of vacuolar phosphate storage forms in plants}

SPX-SLC and SPX-VTC proteins are not encoded in the genomes of later-diverging streptophytes, and SPX-MFS and GIpT/VPE catalyze vacuolar $\mathrm{Pi}$ influx and efflux, respectively, in land plants (Liu et al., 2015, 2016; Xu et al., 2019). We therefore hypothesized that the absence of SPX-SLC and SPX-VTC genes is correlated with the presence of SPX-MFS and GIpT/VPE proteins in the Archaeplastida lineage. Accordingly, we determined the 
A

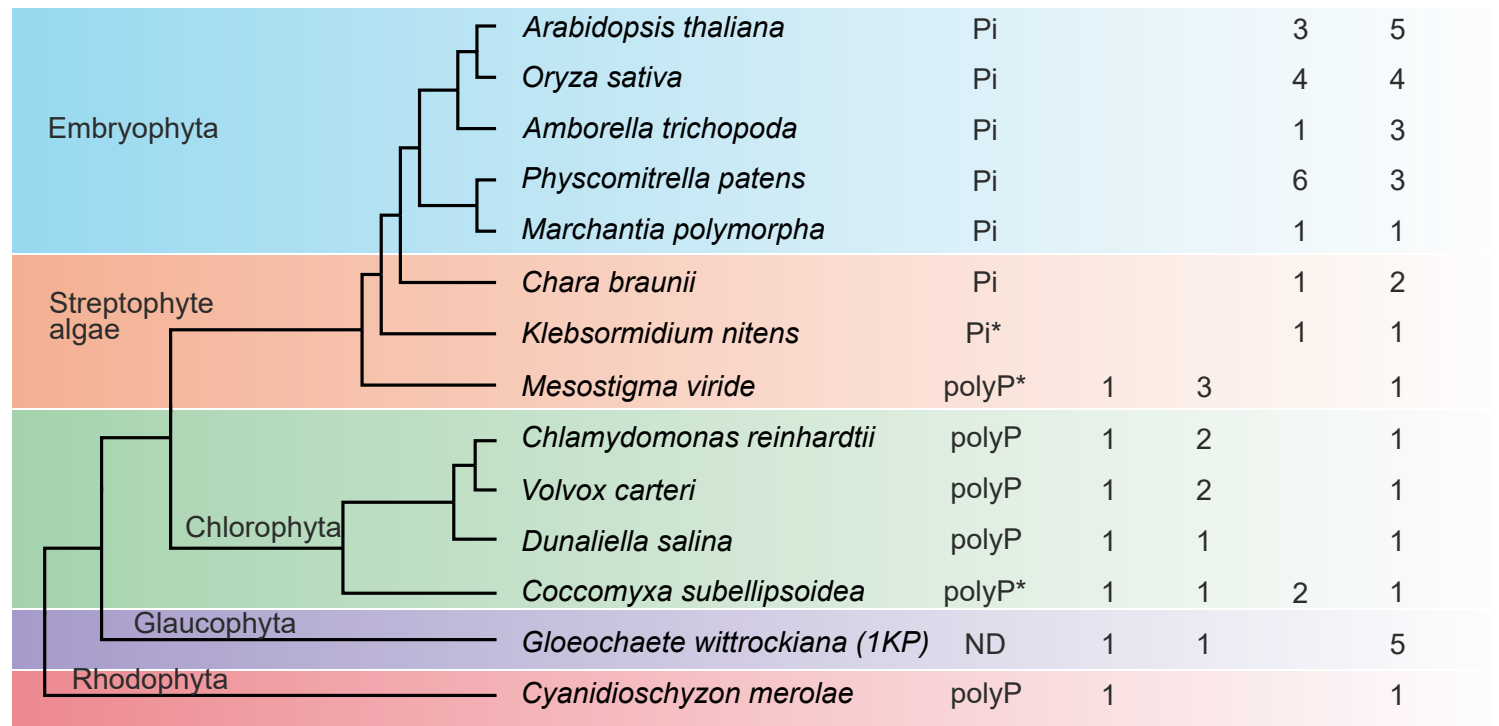

B

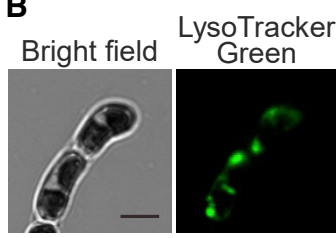

Klebsomidium

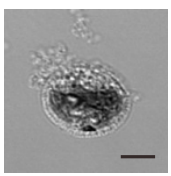

Mesostigma

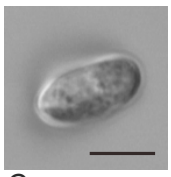

Coccomyxa
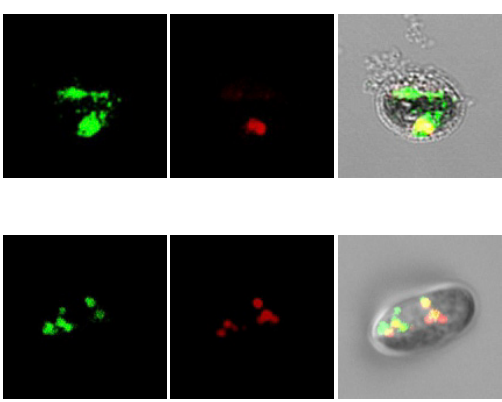

C
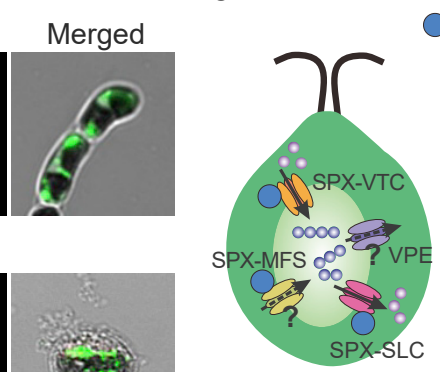

MsPX-sLcs OMspX-vtcs qust

SPX-MFSs MVPEs 000 PolyP oPi

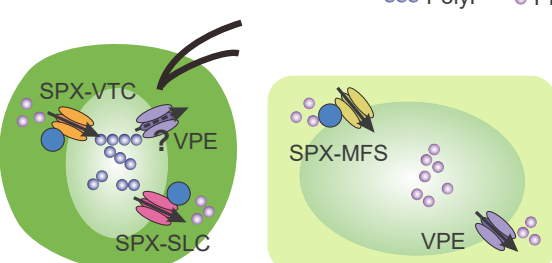

Chlorophyta

Early-diverging
streptophyte $\begin{gathered}\text { Later-diverging Embryophyta } \\ \text { streptophyte }\end{gathered}$

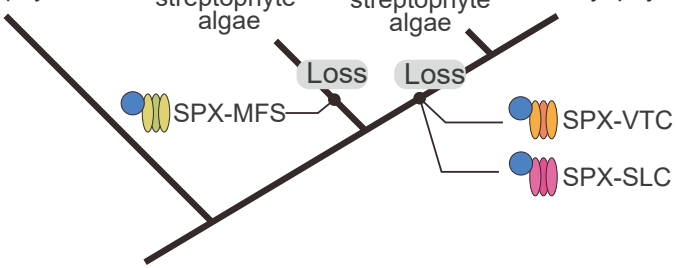

Figure 4. Co-evolution of phosphate transporter and vacuolar phosphorus storage forms in plants.

(A) Identification of the vacuolar phosphorus storage forms and related vacuolar phosphate transporter proteins (SPX-SLC, SPX-VTC, SPX-MFS, and GlpT [VPE]) in representative species from the plant kingdom. The species phylogeny was reconstructed from a concatenation of 154 genome-wide lowcopy orthologous genes. ND, not determined. Asterisk (*) indicates data from this study.

(B) Staining of acidic compartments (LysoTracker Green) and PolyP (DAPI) in algae. Colocalization can be observed in Coccomyxa and Mesostigma but not in Klebsormidium. Scale bars, $5 \mu \mathrm{m}$.

(C) Proposed scenario of the stepwise evolution of SPX-containing genes and vacuolar phosphorus storage forms. The SPX-VTC and SPX-SLC proteins were present in the ancestor of chlorophyte algae and participate in vacuolar polyP synthesis and accumulation, respectively. It is unknown whether GlpT/ VPE and SPX-MFS proteins mediate vacuolar Pi accumulation in the chlorophyte algae. SPX-MFS proteins were lost in early-diverging streptophyte algae. The later-diverging streptophyte algae, which use GIpT/VPE and SPX-MFS for vacuolar Pi homeostasis, have lost SPX-VTC and SPX-SLC proteins, and their vacuolar phosphorus storage form has changed to Pi. Question marks and dashed arrows indicate transporters that lack solid functional identification.

presence of SPX-MFS and GIPT/VPE in species of the green plant lineage (Figure 4A). Genes encoding GlpT/VPE proteins are present in all groups of Viridiplantae, indicating that GIpT/VPE proteins are ancient and conserved among the green plant lineage, but their number increased during the course of embryophyte (land plant) evolution. There are five GIpT/VPE genes in Arabidopsis compared with one in Klebsormidium. SPX-MFS genes are present in the genomes of embryophytes and later-diverging streptophyte algae but absent in the early-diverging unicellular streptophyte Mesostigma. These 


\section{Molecular Plant}

data are consistent with functions of SPX-MFS and GIpT/VPE proteins in vacuolar $\mathrm{Pi}$ homeostasis in late-diverging streptophyte algae and Embryophyta. However, SPX-MFS genes were also found in the genomes of the chlorophytes Asterochloris, Coccomyxa, and Uronema (Supplemental Table 1), which also contain SPX-SLC and SPX-VTC genes. To determine whether the presence of SPX-MFS and GIpT/VPE affects vacuolar phosphorus storage forms, we also examined the presence of vacuolar polyP in Coccomyxa. We showed that Coccomyxa accumulates polyP (Figure 4B). Therefore, these data suggest that polyP is the vacuolar phosphorus storage form in chlorophyte algae that possess SPX-SLC and SPX-VTC genes, despite the presence of GlpT/VPE genes and in some cases the presence of SPX-MFS genes.

These data are consistent with the hypothesis that polyP was the ancestral form of phosphorus storage among the Viridiplantae and that polyP storage required SPX-VTC and SPX-SLC protein functions in the last common ancestor of the Viridiplantae. SPX-VTC and SPX-SLC genes were then lost during early streptophyte evolution, leading to a transition in vacuolar phosphorus storage form from polyP to $\mathrm{Pi}$.

In contrast to polyP, whose production requires ATP synthesis from $\mathrm{Pi}$ (Hothorn et al., 2009), $\mathrm{Pi}$ is an energetically efficient form of vacuolar phosphorus storage in land plants. The fulfillment of cytosolic $\mathrm{Pi}$ demand by vacuolar $\mathrm{Pi}$ is much quicker than by polyP, which must be degraded to Pi before release from the vacuole to the cytosol (Lorenzo-Orts et al., 2020). Given that SPX-MFS and GIpT/VPE genes evolved among later-diverging streptophytes, together with our finding that SPXVTC and SPX-SLC function in chlorophyte vacuolar polyP homeostasis, we propose an evolutionary hypothesis relating the presence of these genes to vacuolar phosphorus storage forms in green plants (Figure 4C). The SPX-VTC and SPX-SLC proteins were present in the ancestor of Chlorophyta algae and participate in vacuolar polyP synthesis and accumulation, respectively. It is unknown whether GIpT/VPE and SPX-MFS proteins mediate vacuolar $\mathrm{Pi}$ accumulation among chlorophyte algae. Although GIpT/VPE and SPX-MFS are both present in certain chlorophytes, their presence is not sufficient to change the major vacuolar phosphorus storage form from polyP to Pi (Figure 4). The later-diverging streptophyte algae have retained GIPT/VPE and SPX-MFS proteins but have lost SPX-VTC and SPX-SLC proteins, and their vacuolar phosphorus storage form has concomitantly changed to $\mathrm{Pi}$. Given that phosphorus storage in the form of $\mathrm{Pi}$ evolved in the aquatic ancestors of land plants and that polyP has not been identified in land plant vacuoles, this transition may be considered a pre-adaptation to life on land. It is formally possible that the transition in vacuolar phosphorus storage form from poly $\mathrm{P}$ to $\mathrm{Pi}$ in Streptophyta may have been adaptive for land colonization.

The gain and loss of genes are two important sources of genetic variation that can cause adaptive phenotypic diversity. There are abundant examples in which increases in gene number are associated with evolutionary changes. For example, Spirogloea muscicola acquired additional genetic capacity through horizontal gene transfer from soil bacteria (Cheng et al., 2019). The genome sequence of Penium margaritaceum reveals an expanded repertoire of genes for cell wall biosynthesis that limit

\section{Evolution of vacuolar P storage forms in green plants}

water loss and protect against UV radiation (Jiao et al., 2020). By contrast, our results suggest that the loss of SPX-SLC and SPX-VTC among later-diverging streptophyte algae and the consequent change in phosphorus storage form from polyP to $\mathrm{Pi}$ may have been a pre-adaptation to land colonization.

\section{METHODS}

\section{C. reinhardtii strains and growth conditions}

The C. reinhardtii WT strain CC-4533 and the mutant strains Crptc11 (LMJ.RY0402.181899), Crptc1-2 (LMJ. RY0402.121135), Crvptc4-1 (LMJ.RY0402.195991), and Crvptc4-2 (LMJ.RY0402.216999) were obtained from the Chlamydomonas Resource Center (Li et al., 2019). Cells were cultured in standard Trisacetate-phosphate (TAP) medium at $\mathrm{pH} 7.0$ under continuous illumination (50 mmol photons $\mathrm{m}^{-2} \mathrm{~s}^{-1}$ ) on a rotating platform (150 rpm) at $24^{\circ} \mathrm{C}$ (Harris, 1989). For transformation, CrPTC1 was cloned into pJM43Ble and transformed into Crptc1-1 by electroporation as previously described (Shimogawara et al., 1998).

\section{Transport activity assay in yeast and oocytes}

The coding regions of $P H O 84, C r P T C 1$, and $P H O 91$ were introduced into the PRS426-ADH1 vector. These constructs and the empty vector were transformed into the yeast strain YP100 (Popova et al., 2010). To determine the localization of CrPTC1, the GFP tag was fused to the N terminus of CrPTC1 and transformed into yeast K699 (Popova et al., 2010). For the transport activity assay in oocytes, full-length cDNA of CrPTC1 was cloned into the oocyte expression vector pT7Ts. The transport activity assay was performed as described previously ( $\mathrm{Xu}$ et al., 2019).

\section{Quantitative real-time PCR analysis}

RNA extraction and expression analysis were performed as described in our previous study (Wang et al., 2020). Primers used for qRT-PCR are listed in Supplemental Table 2.

\section{Measurement of total phosphorus and polyP}

Five milliliters of cells were harvested and dried to measure the total phosphorus concentration as described previously (Chen et al., 2007). PolyP was purified with PCR purification columns (Werner et al., 2005). Purified polyP solution was mixed with an equal volume of $2 \mathrm{M} \mathrm{HCl}$ and heated at $95^{\circ} \mathrm{C}$ for $30 \mathrm{~min}$ for acid degradation, and released $\mathrm{Pi}$ was quantified by the malachite green assay (Ohno and Zibilske, 1991).

\section{Staining PolyP with DAPI}

PolyP within cells was stained with DAPI and imaged using a ZEISS LSM 880 scanning confocal microscope following a previous report (AscharSobbi et al., 2008). Cells were counterstained with $10 \mu \mathrm{M}$ LysoTracker Green for colocalization analysis.

Identification of SPX-containing proteins and construction of the phylogenetic tree

Genome and gene annotation data of 49 selected plant species were obtained from the Phytozome database (Goodstein et al., 2012), NCBI Assembly, PLAZA (Van Bel et al., 2018), and OneKP (One Thousand Plant Transcriptomes Initiative, 2019) (Supplemental Table 1). SPXcontaining proteins were identified by InterProScan 5 (Jones et al., 2014) and validated by BLASTP with an e-value cutoff of $1 e^{-10}$ (Supplemental Table 3). Details of the phylogenetic analysis are provided in Supplemental Document 1. All sequences and information on SPX-containing proteins have been submitted to the Figshare database (https://doi.org/10.6084/m9.figshare.12431861). 
Protein expression and polyP synthesis

Truncated CrVTC4 (amino acids 1-550, CrVTC4p*) was cloned into pET29b (Promega). The $6 \times$ His-CrVTC4p* proteins were purified according to the manufacturer's instructions (Qiagen) and used for polyP synthesis (Hothorn et al., 2009).

\section{SUPPLEMENTAL INFORMATION}

Supplemental information is available at Molecular Plant Online.

\section{FUNDING}

This work was supported by the National Key R\&D Program of China (2017YFD0200200/0200204/0200206). K.Y. was supported by the China Agriculture Research System-Green Manure (CARS-22-G-07) and the Innovation Program of Chinese Academy of Agricultural Sciences. L.D. was supported by the European Research Council Advanced Grant DENOVO-P (contract number 787613).

\section{AUTHOR CONTRIBUTIONS}

K.Y. and Y.Z. conceived and supervised the project. L.W., L.X., Y.Z., and K.Y. designed the research. L.W., YX.Z., and X.J. performed the experiments. L.W., L.X., B.M., X.J., H.Z., HQ.Z., L.D., Y.Z., and K.Y. analyzed the data. L.W., X.J., B.M., L.D., and K.Y. wrote the paper with input from all the authors.

\section{ACKNOWLEDGMENTS}

We thank Dr. Xiaofeng Gu and Dr. Peipei Zhang for M. viride materials, Dr. Wenqiang Yang for supplying the pJM43Ble plasmid, and Dr. J.M. Thevelein for providing the yeast strains. We thank Dr. Yi Wang and Dr. Meiling Zhang for their help with the transport activity assay in oocytes. We also thank Dr. M. Brian Traw for his help with English editing. No conflict of interest declared.

Received: December 27, 2020

Revised: January 20, 2021

Accepted: January 21, 2021

Published: January 27, 2021

\section{REFERENCES}

Aksoy, M., Pootakham, W., and Grossman, A.R. (2014). Critical function of a Chlamydomonas reinhardtii putative polyphosphate polymerase subunit during nutrient deprivation. Plant Cell 26:4214-4229.

Aschar-Sobbi, R., Abramov, A.Y., Diao, C., Kargacin, M.E., Kargacin, G.J., French, R.J., and Pavlov, E. (2008). High sensitivity, quantitative measurements of polyphosphate using a new DAPI-based approach. J. Fluoresc. 18:859-866.

Becker, B., and Marin, B. (2009). Streptophyte algae and the origin of embryophytes. Ann. Bot. 103:999-1004.

Chen, A., Hu, J., Sun, S., and Xu, G. (2007). Conservation and divergence of both phosphate- and mycorrhiza-regulated physiological responses and expression patterns of phosphate transporters in solanaceous species. New Phytol. 173:817-831.

Cheng, S., Xian, W., Fu, Y., Marin, B., Keller, J., Wu, T., Sun, W., Li, X., Xu, Y., Zhang, Y., et al. (2019). Genomes of subaerial Zygnematophyceae provide insights into land plant evolution. Cell 179:1057-1067.e14.

Goodstein, D.M., Shu, S., Howson, R., Neupane, R., Hayes, R.D., Fazo, J., Mitros, T., Dirks, W., Hellsten, U., Putnam, N., et al. (2012). Phytozome: a comparative platform for green plant genomics. Nucleic Acids Res. 40:D1178-D1186.

Hamburger, D., Rezzonico, E., Petétot, J.M.-C., Somerville, C., and Poirier, Y. (2002). Identification and characterization of the Arabidopsis $\mathrm{PHO} 1$ gene involved in phosphate loading to the xylem. Plant Cell 14:889-902.

Harris, E.H. (1989). The Chlamydomonas Sourcebook (Elsevier).
Hothorn, M., Neumann, H., Lenherr, E.D., Wehner, M., Rybin, V., Hassa, P.O., Uttenweiler, A., Reinhardt, M., Schmidt, A., Seiler, J., et al. (2009). Catalytic core of a membrane-associated eukaryotic polyphosphate polymerase. Science 324:513-516.

Jiao, C., Sørensen, I., Sun, X., Sun, H., Behar, H., Alseekh, S., Philippe, G., Palacio Lopez, K., Sun, L., Reed, R., et al. (2020). The Penium margaritaceum genome: hallmarks of the origins of land plants. Cell 181:1097-1111.e2.

Jones, P., Binns, D., Chang, H.-Y., Fraser, M., Li, W., McAnulla, C., McWilliam, H., Maslen, J., Mitchell, A., Nuka, G., et al. (2014). InterProScan 5: genome-scale protein function classification. Bioinformatics 30:1236-1240.

Komine, Y., Eggink, L.L., Park, H., and Hoober, J.K. (2000). Vacuolar granules in Chlamydomonas reinhardtii: polyphosphate and a 70-kDa polypeptide as major components. Planta 210:897-905.

Li, X., Patena, W., Fauser, F., Jinkerson, R.E., Saroussi, S., Meyer, M.T., Ivanova, N., Robertson, J.M., Yue, R., Zhang, R., et al. (2019). A genome-wide algal mutant library and functional screen identifies genes required for eukaryotic photosynthesis. Nat. Genet. $51: 627-635$.

Liu, J., Yang, L., Luan, M., Wang, Y., Zhang, C., Zhang, B., Shi, J., Zhao, F.-G., Lan, W., and Luan, S. (2015). A vacuolar phosphate transporter essential for phosphate homeostasis in Arabidopsis. Proc. Natl. Acad. Sci. U S A 112:E6571-E6578.

Liu, T.-Y., Huang, T.-K., Yang, S.-Y., Hong, Y.-T., Huang, S.-M., Wang, F.-N., Chiang, S.-F., Tsai, S.-Y., Lu, W.-C., and Chiou, T.-J. (2016). Identification of plant vacuolar transporters mediating phosphate storage. Nat. Commun. 7:11095.

Lorenzo-Orts, L., Couto, D., and Hothorn, M. (2020). Identity and functions of inorganic and inositol polyphosphates in plants. New Phytol. 225:637-652.

Lv, Q., Zhong, Y., Wang, Y., Wang, Z., Zhang, L., Shi, J., Wu, Z., Liu, Y., Mao, C., Yi, K., et al. (2014). SPX4 negatively regulates phosphate signaling and homeostasis through its interaction with PHR2 in rice. Plant Cell 26:1586-1597.

Nie, Y., Foster, C.S.P., Zhu, T., Yao, R., Duchêne, D.A., Ho, S.Y.W., and Zhong, B. (2020). Accounting for uncertainty in the evolutionary timescale of green plants through clock-partitioning and fossil calibration strategies. Syst. Biol. 69:1-16.

Ohno, T., and Zibilske, L.M. (1991). Determination of low concentrations of phosphorus in soil extracts using malachite green. Soil Sci. Soc. Am. J. 55:892-895.

One Thousand Plant Transcriptomes Initiative. (2019). One thousand plant transcriptomes and the phylogenomics of green plants. Nature 574:679-685.

Park, B.S., Seo, J.S., and Chua, N.-H. (2014). NITROGEN LIMITATION ADAPTATION recruits PHOSPHATE2 to target the phosphate transporter PT2 for degradation during the regulation of Arabidopsis phosphate homeostasis. Plant Cell 26:454-464.

Pick, U., and Weiss, M. (1991). Polyphosphate hydrolysis within acidic vacuoles in response to amine-induced alkaline stress in the halotolerant alga Dunaliella salina. Plant Physiol. 97:1234-1240.

Poirier, Y., Thoma, S., Somerville, C., and Schiefelbein, J. (1991). Mutant of Arabidopsis deficient in xylem loading of phosphate. Plant Physiol. 97:1087-1093.

Popova, Y., Thayumanavan, P., Lonati, E., Agrochão, M., and Thevelein, J.M. (2010). Transport and signaling through the phosphate-binding site of the yeast Pho84 phosphate transceptor. Proc. Natl. Acad. Sci. U S A 107:2890-2895.

Puga, M.I., Mateos, I., Charukesi, R., Wang, Z., Franco-Zorrilla, J.M., de Lorenzo, L., Irigoyen, M.L., Masiero, S., Bustos, R., Rodriguez, J., et al. (2014). SPX1 is a phosphate-dependent inhibitor of 


\section{Molecular Plant}

PHOSPHATE STARVATION RESPONSE 1 in Arabidopsis. Proc. Natl. Acad. Sci. U S A 111:14947-14952.

Raghothama, K.G. (1999). Phosphate acquisition. Annu. Rev. Plant Physiol. Plant Mol. Biol. 50:665-693.

Ruiz, F.A., Marchesini, N., Seufferheld, M., Govindjee, and Docampo, R. (2001). The polyphosphate bodies of Chlamydomonas reinhardtii possess a proton-pumping pyrophosphatase and are similar to acidocalcisomes. J. Biol. Chem. 276:46196-46203.

Shimogawara, K., Fujiwara, S., Grossman, A., and Usuda, H. (1998). High-efficiency transformation of Chlamydomonas reinhardtii by electroporation. Genetics 148:1821-1828.

Van Bel, M., Diels, T., Vancaester, E., Kreft, L., Botzki, A., Van de Peer, Y., Coppens, F., and Vandepoele, K. (2018). Plaza 4.0: an integrative resource for functional, evolutionary and comparative plant genomics. Nucleic Acids Res. 46:D1190-D1196.

Wang, Z., Ruan, W., Shi, J., Zhang, L., Xiang, D., Yang, C., Li, C., Wu, Z., Liu, Y., Yu, Y., et al. (2014). Rice SPX1 and SPX2 inhibit phosphate starvation responses through interacting with PHR2 in a phosphatedependent manner. Proc. Natl. Acad. Sci. U S A 111:14953-14958.
Evolution of vacuolar $P$ storage forms in green plants

Wang, L., Xiao, L., Yang, H., Chen, G., Zeng, H., Zhao, H., and Zhu, Y. (2020). Genome-wide identification, expression profiling, and evolution of phosphate transporter gene family in green algae. Front. Genet. 11. https://doi.org/10.3389/fgene.2020.590947.

Werner, T.P., Amrhein, N., and Freimoser, F.M. (2005). Novel method for the quantification of inorganic polyphosphate (iPoP) in Saccharomyces cerevisiae shows dependence of IPoP content on the growth phase. Arch. Microbiol. 184:129-136.

Wykoff, D.D., Grossman, A.R., Weeks, D.P., Usuda, H., and Shimogawara, K. (1999). Psr1, a nuclear localized protein that regulates phosphorus metabolism in Chlamydomonas. Proc. Natl. Acad. Sci. U S A 96:15336-15341.

Xu, L., Zhao, H., Wan, R., Liu, Y., Xu, Z., Tian, W., Ruan, W., Wang, F., Deng, M., Wang, J., et al. (2019). Identification of vacuolar phosphate efflux transporters in land plants. Nat. Plants 5:84-94.

Yagisawa, F., Nishida, K., Yoshida, M., Ohnuma, M., Shimada, T., Fujiwara, T., Yoshida, Y., Misumi, O., Kuroiwa, H., and Kuroiwa, T. (2009). Identification of novel proteins in isolated polyphosphate vacuoles in the primitive red alga Cyanidioschyzon merolae. Plant J. 60:882-893. 